\title{
OBJETO DE APRENDIZAJE PARA LOS ODS EN INGLÉS Y ESPAÑOL CON REPRESENTACIONES SEMÁNTICAS
}

\section{LEARNING OBJECT FOR THE SDGs IN ENGLISH AND SPANISH WITH SEMANTIC REPRESENTATIONS}

\author{
María Auxilio Medina Nieto*, Delia Arrieta Díaz** \\ Araceli Ortíz Carranco***
}

\begin{abstract}
*Doctora en Ciencias de la Computación. Universidad Politécnica de Puebla. Departamento de Posgrado. ORCID: http://orcid.org/0000-0001-6391-4799.

**Doctora en Gobierno y Administración Pública. Facultad de Economía, Contaduría y Administración de la Universidad Juárez del Estado de Durango. Email: darrietad@hotmail.com. ORCID: http://orcid.org/00000001-7239-3761.

***Doctora en Administración. Universidad Politécnica de Puebla. Ingeniería Financiera. Email: araceli.ortiz@uppuebla.edu.mx. ORCID: http://orcid.org/0000-0001-7835-6339.
\end{abstract}

Dirección para recibir correspondencia: maria.medina@uppuebla.edu.mx; mauxmedina@gmail.com

Fecha de recibido: 19 de septiembre de 2019

Fecha de aceptación: 23 de noviembre de 2019 
OBJETO DE APRENDIZAJE PARA LOS ODS EN INGLÉS Y ESPAÑOL CON REPRESENTACIONES SEMÁNTICAS.

\section{RESUMEN}

El artículo presenta un objeto de aprendizaje diseñado para introducir la temática de los Objetivos de Desarrollo Sostenible (ODS) de la Agenda 2030 de la Organización de las Naciones Unidas; los contenidos se extraen del tesauro y las páginas web en inglés y español de esa organización.

OBJETIVO: Apoyar la adquisición de un vocabulario común en ambos idiomas que permita a los estudiantes alcanzar un nivel de conocimiento básico sobre los ODS. El vocabulario se emplea en mapas conceptuales, grafos y un modelo semántico u ontología.

MATERIAL Y MÉTODO: La construcción del objeto de aprendizaje se realizó conforme a la metodología ADDIE, siglas que corresponden a las fases de: análisis, diseño, desarrollo, implementación y evaluación. Esta metodología es utilizada ampliamente para el desarrollo de objetos de aprendizaje dirigidos a estudiantes de nivel medio superior, superior y posgrado.

RESULTADOS: Se describen los resultados de cada fase de la metodología ADDIE. Para la evaluación, se llevó a cabo un estudio exploratorio dirigido a estimar la percepción de estudiantes o usuarios potenciales; las métricas empleadas corresponden a la escala de usabilidad y registro de red de promotores, ambas se emplean frecuentemente en la literatura relacionada con evaluaciones heurísticas de las Tecnologías de la Información y la Comunicación (TIC). Los valores obtenidos fueron 88.6 y 8.1, respectivamente, en ambos casos, indican un grado de percepción alto.

CONCLUSIONES: El objeto de aprendizaje propuesto representa una aplicación de las tecnologías semánticas y las TIC, dirigida a atender necesidades de formación básica para la sostenibilidad.

PALABRAS CLAVE: Desarrollo sostenible. Objetivos de Desarrollo Sostenible. Objetos de aprendizaje. Tecnologías de la Información y la Comunicación. Tecnologías semánticas. 
OBJETO DE APRENDIZAJE PARA LOS ODS EN INGLÉS Y ESPAÑOL CON REPRESENTACIONES SEMÁNTICAS.

\section{ABSTRACT}

The article presents a learning object designed to introduce the theme of the Sustainable Development Goals (SDGs) of the 2030 Agenda of the United Nations Organization; the contents are extracted from the thesaurus and the web pages in English and Spanish of that organization.

OBJECTIVE: Support the acquisition of a common vocabulary in both languages that allows students to reach a level of basic knowledge about the SDGs. The vocabulary is used in concept maps, graphs and a semantic model or ontology

MATERIAL AND METHOD: The construction of the learning object was carried out according to the ADDIE methodology, acronym that corresponds to the phases of analysis, design, development, implementation and evaluation. This methodology is widely used for the development of learning objects aimed at middle-upper, upper and postgraduate students.

RESULTS: The results of each phase of the ADDIE methodology are described. For the evaluation, an exploratory study was carried out to estimate the perception of potential students or users; the metrics used correspond to the scale of usability and network registration of promoters, both are frequently used in the literature related to heuristic evaluations of Information and Communication Technologies (ICT). The values obtained were 88.6 and 8.1, respectively, in both cases, they indicate a high degree of perception.

CONCLUSIONS: The proposed learning object represents an application of the semantic technologies and the ICT, aimed to respond to the basic training needs for sustainability.

KEYWORDS: Sustainable development. Sustainable Development Goals. Learning objects. Information and Communication Technologies. Semantic technologies.

\section{INTRODUCCIÓN}

El Desarrollo Sostenible (DS) de la Agenda 2030 de la Organización de las Naciones Unidas (ONU) es "Una visión compartida, holística y a largo plazo que los países han acordado como el mejor camino para mejorar la vida de las personas en todo el mundo" (United Nations, 2015); es un modelo de desarrollo que busca satisfacer las necesidades del presente sin comprometer la capacidad de las generaciones futuras (Brundtland et. al., 1987), (Arboleda, 2016). 
OBJETO DE APRENDIZAJE PARA LOS ODS EN INGLÉS Y ESPAÑOL CON REPRESENTACIONES SEMÁNTICAS.

EI DS y los Objetivos de Desarrollo Sostenible (ODS) son temas abordados en los diferentes programas académicos de la Universidad Politécnica de Puebla (UPPue) y la Universidad Juárez del Estado de Durango (UJED), a través de diálogos con los estudantes, se observó que entrevistas con entrevistas, los estudantes señalaron como fuentes de consulta las páginas web de la ONU, (ODS 2019), (SDG 2019). Desde el punto de vista de las autoras, la problemática detectada consiste en que en esas páginas existen diferencias tanto en los aspectos de la interfaz como en la profundidad y nivel de detalle de los contenidos, lo que da como resultado que los estudiantes que sólo revisan los contenidos en español acceden a menor información que la distribuida en el idioma inglés. Para atender esa problemática, el artículo propone la construcción de un objeto de aprendizaje (OA) con base en la metodología ADDIE, siglas que corresponden al Análisis, Diseño, Desarrollo, Implementación y Evaluación (Branch, 2009), que integra información de ambas páginas y la representa en mapas conceptuales y grafos, el objetivo es apoyar la adquisición de un vocabulario común necesario para alcanzar un nivel de conocimientos básico sobre los ODS.

\section{MATERIAL Y MÉTODO}

En el contexto internacional, la ONU disemina información a través de documentos distribuidos bajo políticas de acceso abierto (AA) almacenados en repositorios como los descritos en la Tabla 1 (DOAR 2019). Una descripción extendida de los ODS en español está disponible en el sitio web para la Alimentación y la Agricultura (FAO) (FAO, 2019), un conjunto de videos que explican los ODS se reporta en (Murillo, 2018).

La versión en español e inglés de las páginas web de la ONU, (ODS 2019) y (SDG 2019) respectivamente, son referencias fundamentales que describen qué es el DS y cuáles son los 17 ODS. A partir de un análisis del contenido y su presentación, se cuenta con una organización, uso de íconos y texto similar. Sin embargo, se observaron las diferencias siguientes:

- En la versión en inglés, el título es una imagen que incluye al logotipo de la ONU, el círculo que hace referencia a los ODS y los términos "Sustainable development goals"; en español, el título sólo está en texto.

- En la versión en español, la descripción de los ODS se despliega al pasar el ratón sobre los íconos; es decir, en "tool tips", en tanto, en la versión en inglés, estas descripciones forman 
OBJETO DE APRENDIZAJE PARA LOS ODS EN INGLÉS Y ESPAÑOL CON REPRESENTACIONES SEMÁNTICAS.

parte de las propias imágenes, lo cual no permite que el texto sea reutilizado en otro documento o procesado de forma automática.

\section{Tabla 1}

Repositorios de la ONU

\begin{tabular}{lccc}
\hline Nombre del repositorio & Tipo & País & $\begin{array}{c}\text { Idioma de los } \\
\text { documentos }\end{array}$ \\
\hline FAO Document Repository & Institucional & Italia & $\begin{array}{c}\text { Árabe, inglés, francés, } \\
\text { español }\end{array}$ \\
MOST Digital Library & Institucional & Francia & Inglés \\
Majaliss & Disciplinario & Francia & Árabe \\
United Nations Digital Library Islamabad & Institucional & Estados Unidos & Inglés \\
World Digital Library & Gobierno & Francia & Inglés, árabe, chino, \\
& & & francés
\end{tabular}

Fuente: (DOAR, 2019).

- En la versión en inglés, las imágenes que describen a los ODS contienen enlaces, técnicamente, vínculos o direcciones de internet, que acceden a otras páginas que incluyen una descripción de mayor longitud comparada con la versión en español, así como una tabla que describe a cada uno de los 17 ODS mediante las siguientes columnas: 1) hechos y figuras, 2) metas y 3) otros enlaces.

- Una descripción del objetivo 12 para la versión en inglés es "Recycle paper, plastic, glass \& aluminium", que está en una imagen, sin embargo, otra distinta se muestra al reverso, ésta última coincide con el título del propio objetivo.

En general, en términos de número de caracteres y número de imágenes utilizadas, entre otros elementos, existe mayor información para cada ODS en la página en inglés en comparación con la de español.

De forma alternativa, para localizar fuentes de información validadas que traten la temática de los ODS, se utilizó el Repositorio Nacional (RN), el cual, a la fecha de elaboración de este artículo, integra datos de 100 Repositorios Institucionales (RIs) que pertenecen a universidades y centros de investigación de México, los RIs distribuyen contenidos académicos y científicos bajo los términos de políticas de acceso abierto (AA), esto implica que el formato es digital, el acceso es 
OBJETO DE APRENDIZAJE PARA LOS ODS EN INGLÉS Y ESPAÑOL CON REPRESENTACIONES SEMÁNTICAS.

en línea, que están libres de cargo y de restricciones de licencias y derechos de autor (Suber, 2011). De acuerdo con (Chen, 2013), entre los beneficios del AA se encuentran los siguientes: 1) democratización de información, 2) incremento en la posibilidad de que la información influencie a políticas públicas y 3 ) visibilidad de documentos, autores y organizaciones.

Utilizando la interfaz de búsqueda avanzada del RN, se introdujeron las palabras clave "objetivos" y "desarrollo sostenible" y se recuperaron 1517 documentos que corresponden a 884 tesis de maestría, 244 artículos, 174 tesis de doctorado, 94 capítulos de libro y 69 libros, en los cuales las palabras clave aparecen en los datos descriptivos pero no todas en los títulos.

La diversidad, profundidad y número de fuentes de información que abordan la temática de los ODS, así como las diferencias entre las páginas en inglés y español de la ONU, motivaron la construcción del OA de este artículo, denominado Onto4ODS, acrónimo de "ontología o modelo semántico para los ODS". La construcción de Onto4ODS se realizó conforme al modelo instruccional o metodología ADDIE, ésta integra las etapas de Análisis, Diseño, Desarrollo, Implementación y Evaluación, (Branch, 2009). Los resultados de estas etapas se presentan en la sección 3.

\section{RESULTADOS}

Un OA es un tipo de recurso educativo abierto, corresponde a una entidad digital o no digital que se utiliza durante el aprendizaje apoyado con tecnología (IEEE, 2019). Un OA integra elementos tecnológicos y pedagógicos, tales como: objetivo de aprendizaje, materiales didácticos o contenidos, diseño instruccional, actividades de aprendizaje y evaluación, así como datos descriptivos o metadatos. Los materiales se distribuyen en formatos como videos, páginas web, audios, textos, animaciones e imágenes. La tabla 2, muestra los resultados del análisis para Onto4ODS; notar que el dominio de aprendizaje es cognoscitivo, dado que se relaciona con procesos o habilidades del pensamiento.

Los resultados de la tabla 2, sirven como base para la segunda etapa, el diseño, el cual se resume en la tabla 3; en esta tabla, considerar que el verbo "seleccionar" del objetivo instruccional se ubica en el nivel de "comprensión" de acuerdo con la taxonomía de Bloom (Gogus, 2012). A través de herramientas tecnológicas como cuestionarios o bancos de preguntas, se recolectan los datos que evidencian el cumplimiento o no de esta acción de manera automática. 
OBJETO DE APRENDIZAJE PARA LOS ODS EN INGLÉS Y ESPAÑOL CON REPRESENTACIONES SEMÁNTICAS.

\section{Tabla 2}

Resultado de la etapa de análisis

\begin{tabular}{|c|c|}
\hline Nombre del OA: & Onto4ODS \\
\hline Preguntas generadoras & $\begin{array}{l}\text { ¿Qué es el DS?, ¿por qué es importante conocer los ODS?, ¿qué } \\
\text { términos en inglés y español describen a los ODS? }\end{array}$ \\
\hline Créditos & $\begin{array}{l}\text { María Auxilio Medina Nieto }{ }^{1} \text {, Delia Arrieta Díaz }{ }^{2} \text {, Araceli Ortiz } \\
\text { Carranco }^{1} \text {. }\end{array}$ \\
\hline Instituciones de las autoras & $\begin{array}{l}\text { Universidad Politécnica de Puebla }{ }^{1} \text {. } \\
\text { Universidad Juárez del Estado de Durango }{ }^{2} \text {. }\end{array}$ \\
\hline Perfil de usuarios & Estudiantes de licenciatura. \\
\hline $\begin{array}{l}\text { Habilidades, preferencias y } \\
\text { actitudes }\end{array}$ & $\begin{array}{l}\text { Uso de TIC, internet, autodidacta, nivel de inglés básico o } \\
\text { intermedio, manejo intermedio de computadora. }\end{array}$ \\
\hline Necesidad formativa & $\begin{array}{l}\text { Identificar los términos que describen al DS y de los ODS en inglés } \\
\text { y español. }\end{array}$ \\
\hline Ámbito de interés & $\begin{array}{l}\text { Revisión de documentos en inglés y español que versen sobre el } \\
\text { DS y los ODS. }\end{array}$ \\
\hline Derechos de autor & $\begin{array}{l}\text { Acceso abierto. Licencia: Creative Commons CC-BC-NC-ND } 2.5 \\
\text { MX. }\end{array}$ \\
\hline Limitaciones & $\begin{array}{l}\text { La descripción de los ODS se muestra en grafos, mapas } \\
\text { conceptuales y en un modelo semántico, en inglés y español, no } \\
\text { incluye la definición de indicadores ni de las } 169 \text { metas. }\end{array}$ \\
\hline
\end{tabular}

Fuente: Elaboración propia. 
OBJETO DE APRENDIZAJE PARA LOS ODS EN INGLÉS Y ESPAÑOL CON REPRESENTACIONES SEMÁNTICAS.

\section{Tabla 3}

Resultado de la etapa de diseño

\begin{tabular}{|c|c|}
\hline Objetivo instruccional & $\begin{array}{l}\text { El estudiante seleccionará la traducción al inglés de la descripció } \\
\text { de los ODS en un cuestionario con un mínimo de } 70 \% \text { de aciertos }\end{array}$ \\
\hline Contenido & $\begin{array}{l}\text { 1. Definición de DS } \\
\text { 2. Identificación de los } 17 \text { ODS } \\
\text { 3. Descripción de los } 17 \text { ODS con términos en inglés y español }\end{array}$ \\
\hline Grado de dificultad & Básico \\
\hline Actividad de aprendizaje & $\begin{array}{l}\text { Revisión de mapas conceptuales y grafos, ejercicios de bancos d } \\
\text { palabras }\end{array}$ \\
\hline Actividades de evaluación & Cuestionario de 5 preguntas con opción múltiple (3 opciones) \\
\hline Recursos de uso & Navegador, internet \\
\hline Recursos de desarrollo & $\begin{array}{l}\text { Lenguaje HTML5, editor de ontologías Protégé (Musen, 2015) } \\
\text { módulo OntoGraph, Adobe Reader, CmapTools (Novak \& Cañas } \\
\text { 2008), Power Point de Microsoft. }\end{array}$ \\
\hline Tiempo de dedicación & $\begin{array}{l}90 \text { minutos } \\
\text { 1. http://www.onu.org.mx/agenda-2030/objetivos-del-desarrollo- } \\
\text { sostenible/ } \\
\text { 2. https://lib-thesaurus.un.org/LIB/DHLUNBISThesaurus.nsf/ }\end{array}$ \\
\hline Enlaces relacionados & $\begin{array}{l}\text { 3. https://www.un.org/sustainabledevelopment/sustainable- } \\
\text { development-goals/ }\end{array}$ \\
\hline
\end{tabular}

Fuente: Elaboración propia.

Durante las etapas de desarrollo e implementación se elaboraron los contenidos de Onto4ODS utilizando los recursos descritos en la tabla 3. A manera de ejemplo, la figura 1 muestra el mapa conceptual que describen los términos relacionados con DS de acuerdo con el tesauro de la ONU (UNBIS, 2019). Es importante resaltar, que la barra con íconos al final de la figura 1 se distribuye por la ONU bajo los términos del AA. 
OBJETO DE APRENDIZAJE PARA LOS ODS EN INGLÉS Y ESPAÑOL CON REPRESENTACIONES SEMÁNTICAS.

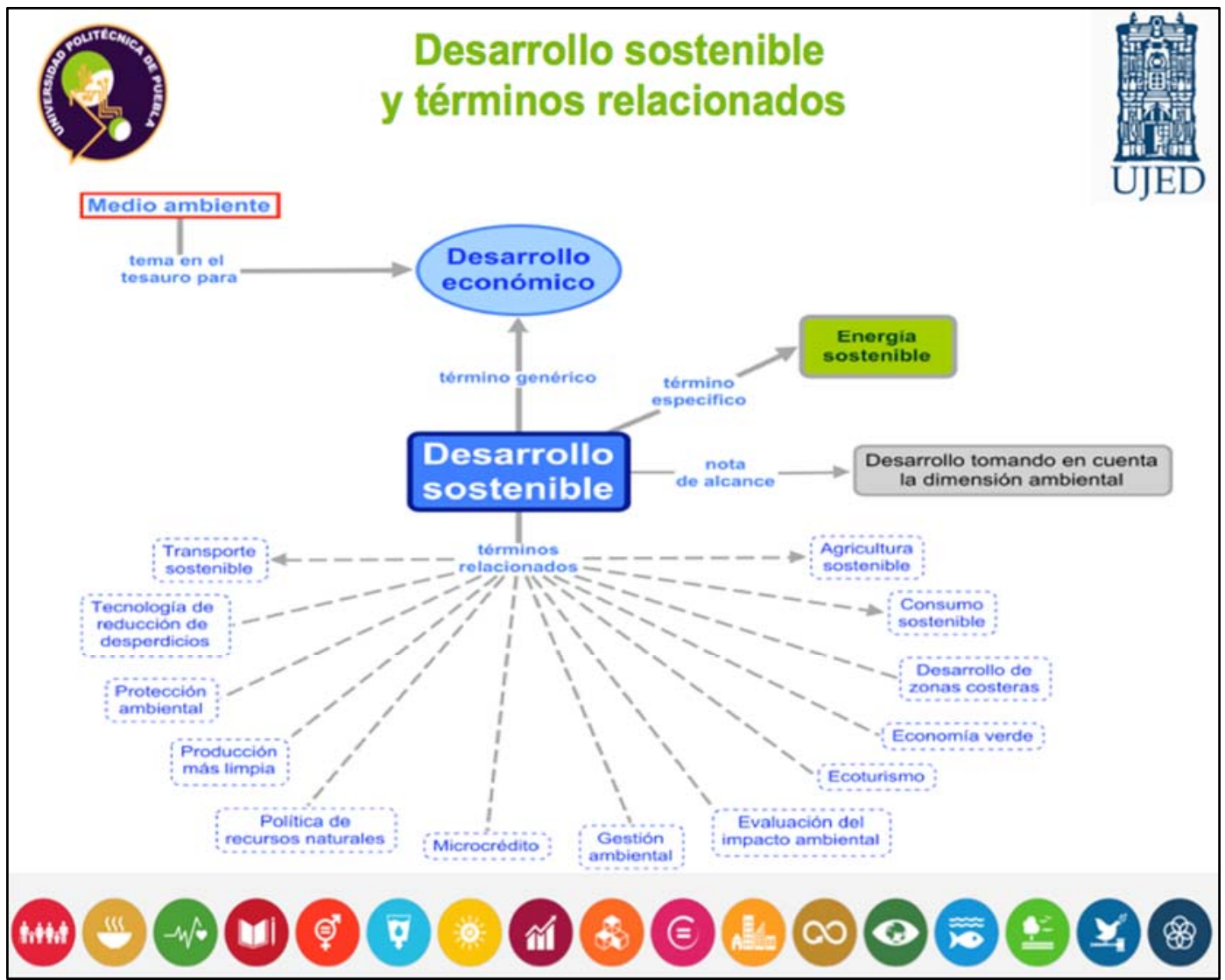

Figura 1. Mapa semántico del tesauro de la ONU para DS.

Fuente: Elaboración propia.

Al utilizar la opción "Propositions" y el botón "Export as Text" de CmapTools, la información del mapa de la figura 1 , se transforma en un conjunto de proposiciones que son validadas por expertos, algunas de éstas se aprecian en la figura 2.

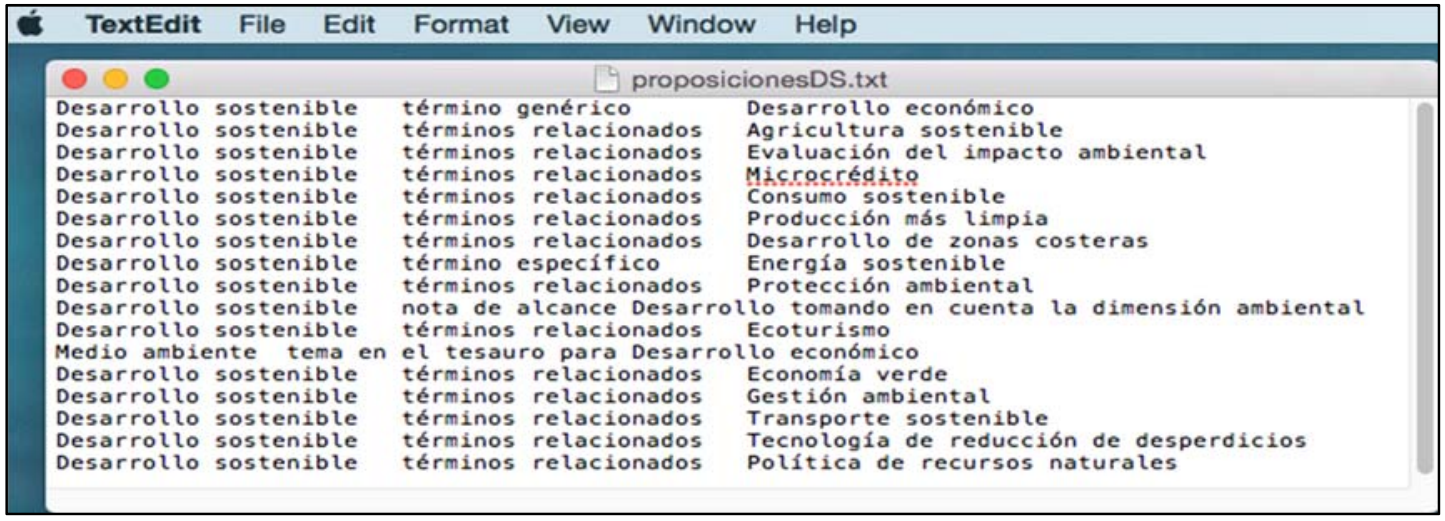

Figura 2. Proposiciones del mapa semántico de la figura 1.

Fuente: Elaboración propia. 
OBJETO DE APRENDIZAJE PARA LOS ODS EN INGLÉS Y ESPAÑOL CON REPRESENTACIONES SEMÁNTICAS.

La figura 3, muestra la versión en inglés de la figura 1; notar que el idioma se muestra en el rectángulo de la esquina superior izquierda.

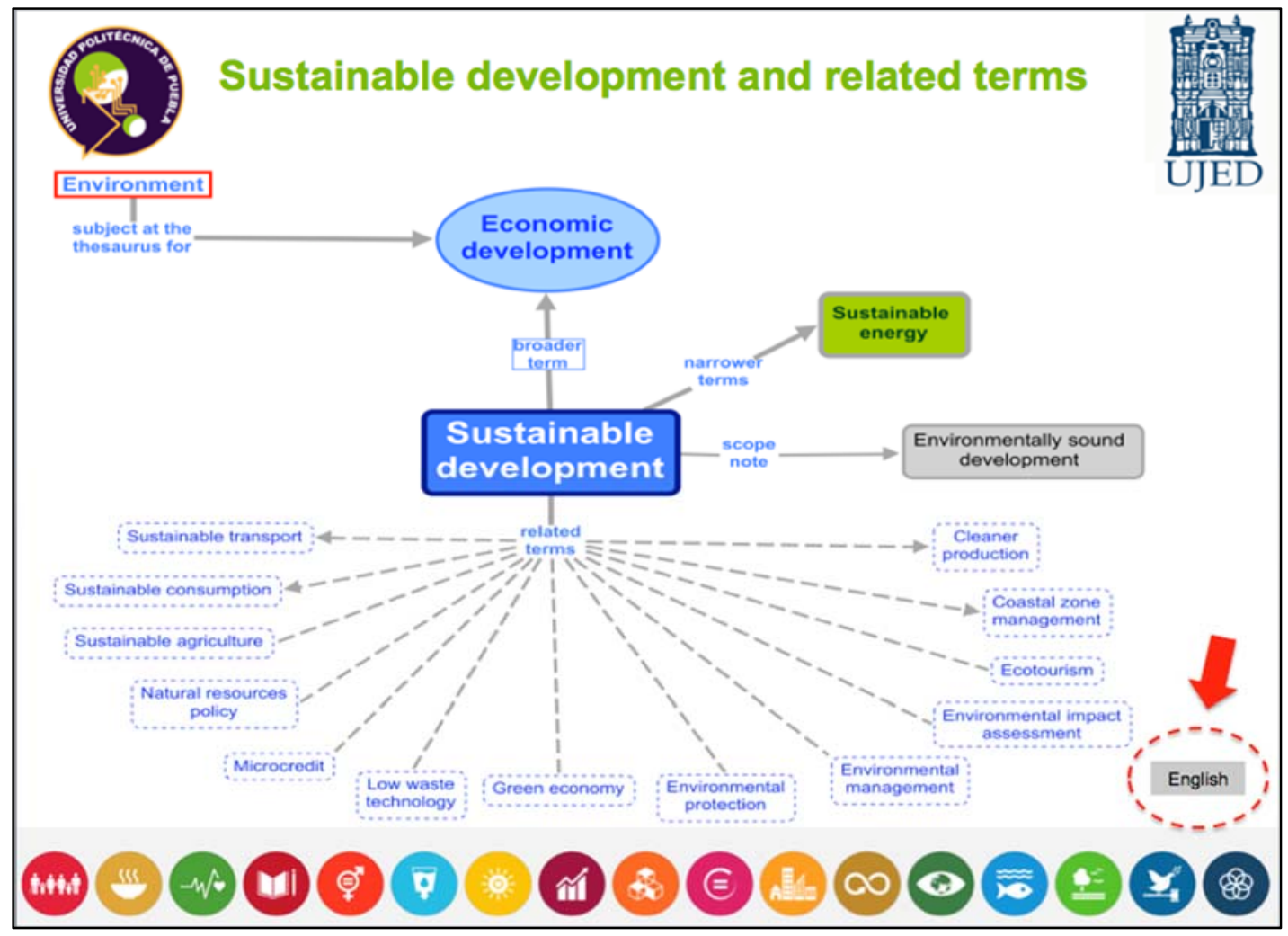

Figura 3. Mapa semántico del tesauro de la ONU para DS, versión en idioma inglés. Fuente: Elaboración propia.

La figura 4, muestra 26 términos agrupados por clase e idioma provenientes del tesauro de la ONU, (UNBIS, 2019), éstos se integran al modelo semántico de Onto4ODS desarrollado en el editor Protégé (Musen, 2015). Las traducciones se encuentran en las columnas "seTraduceComo" e "isTranslatedAs".

El modelo semántico incluye el nombre y la descripción de cada uno de los 17 ODS, esta información se representa en grafos como el de la figura 5. Adicionalmente, en Protégé, se puede utilizar un mecanismo de búsqueda para recuperar información a partir de la introducción de una palabra clave. Por ejemplo, la figura 5 muestra el resultado de buscar un ODS cuya descripción contenga la palabra "desperdicio". Observe que la información se despliega en inglés y español al pasar el ratón sobre los elementos del grafo. 
OBJETO DE APRENDIZAJE PARA LOS ODS EN INGLÉS Y ESPAÑOL CON REPRESENTACIONES SEMÁNTICAS.

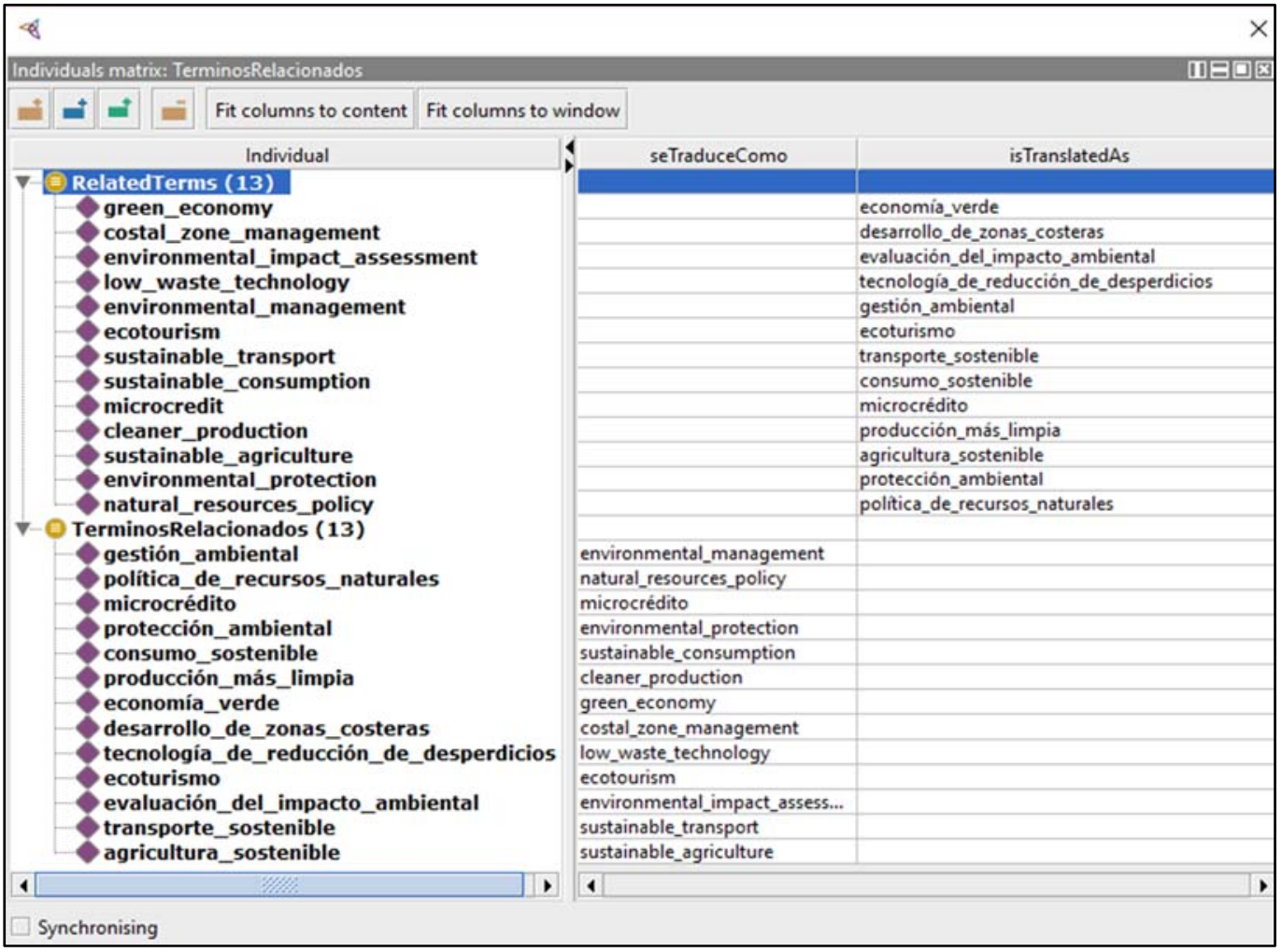

Figura 4. Traducción de términos relacionados con DS.

Fuente: Elaboración propia.

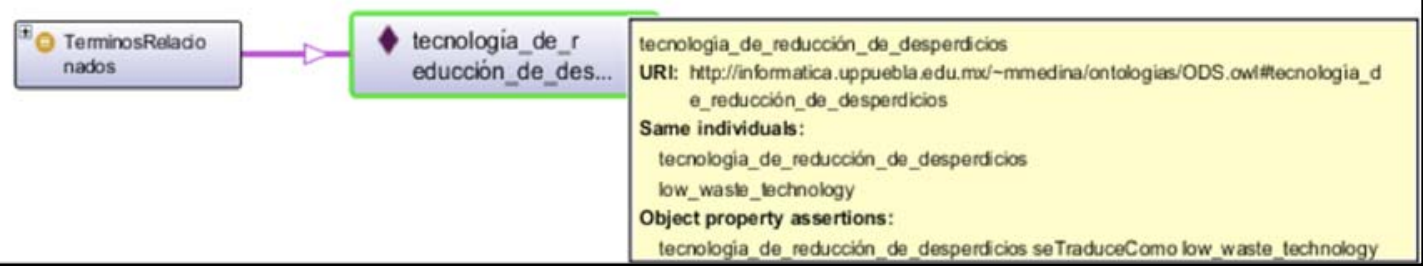

Figura 5. Recuperación de una descripción a partir de una palabra clave.

Fuente: Elaboración propia.

El modelo semántico emplea un lenguaje formal que permite a usuarios y computadoras acceder a la información a través de tecnologías semánticas. A manera de ilustración, la figura 6 muestra una consulta en el lenguaje SPARQL (parte superior) y su resultado, en este caso, consiste en la descripción en inglés y español de los ODS 1, 10 y 11, (parte inferior). 
OBJETO DE APRENDIZAJE PARA LOS ODS EN INGLÉS Y ESPAÑOL CON REPRESENTACIONES SEMÁNTICAS.

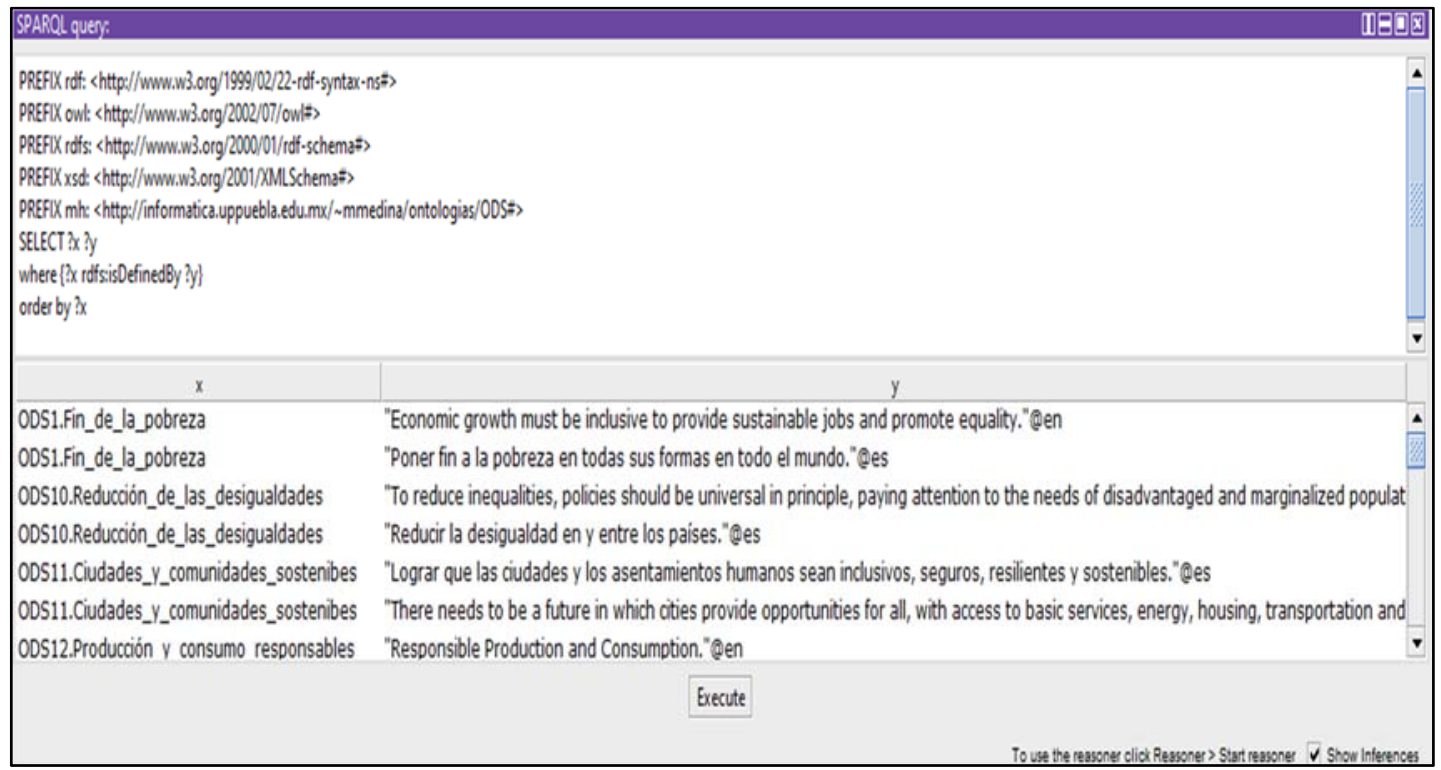

Figura 6. Recuperación de descripciones con tecnologías semánticas.

Fuente: Elaboración propia.

Entre los beneficios de utilizar las tecnologías semánticas, resalta que a través de programas de software denominados razonadores o clasificadores, se verifica automáticamente la consistencia lógica del modelo semántico. La Sección 4 muestra los resultados de la etapa de evaluación.

\section{DISCUSIÓN}

En la etapa de evaluación, se llevó a cabo un estudio exploratorio dirigido a estimar la percepción de uso de Onto4ODS por parte de usuarios potenciales; se formaron los grupos de usuarios descritos en la tabla 4; los participantes se eligieron a través de un método aleatorio, forman parte de la comunidad universitaria de la UPPue. 
OBJETO DE APRENDIZAJE PARA LOS ODS EN INGLÉS Y ESPAÑOL CON REPRESENTACIONES SEMÁNTICAS.

Tabla 4

Descripción de participantes de pruebas

\begin{tabular}{cccc}
\hline & Rol & Género & Edad (años) \\
\hline Grupo A & Docentes: $\underline{5}$ & Femenino: $\underline{3}$ & Entre 25 y 35: $\underline{0}$ \\
& & Masculino: $\underline{2}$ & Entre 36 y 45: $\underline{3}$ \\
& & & \\
& & & \\
Grus de 45: $\underline{2}$ \\
& Estudiante de & & Entre 18 y 21: $\underline{7}$ \\
& licenciatura: $\underline{7}$ & Femenino: $\underline{5}$ & Entre 22 y 25: $\underline{2}$ \\
& Estudiante de & Masculino: $\underline{5}$ & Más de 25: $\underline{1}$ \\
\hline
\end{tabular}

Fuente: Elaboración propia.

El estudio se diseñó conforme al protocolo descrito en (Nielsen, 1997); los pasos implementados por sesión fueron:

1. La evaluadora entregó la carta de derechos a cada participante.

2. La evaluadora solicitó al participante su firma en el formato de consentimiento.

3. El participante contestó un cuestionario para indagar sobre la experiencia de uso de OAs.

4. La evaluadora solicitó al participante realizar las tareas siguientes: 1) búsqueda y recuperación de la definición de DS en inglés y 2) descripción del ODS No. 4: Educación en español. El participante explicó sus acciones, decisiones y opiniones mientras revisaba los contenidos de Onto4ODS para ejecutar las tareas; es decir, implementó la modalidad conocida como verbalización individual

5. La evaluadora recolectó las opiniones de cada participante en un instrumento formado por los componentes siguientes (Muñoz, González y Sánchez, 2014):

a) Escala de usabilidad de sistema, (SUS de las siglas en inglés de System Usability Scale), cuestionario estandarizado de diez preguntas que obtiene una medida cuantitativa de aceptabilidad y usabilidad; un resultado menor a 68 indica que el objeto de prueba no es aceptable (Brooke, 2013). 
OBJETO DE APRENDIZAJE PARA LOS ODS EN INGLÉS Y ESPAÑOL CON REPRESENTACIONES SEMÁNTICAS.

b) Net Promoter Score (NPS), pregunta estándar que se emplea para estimar actitudes de los participantes en relación al objeto de prueba (Fessenden, 2016). Se pide una respuesta numérica entre 0 y 10 , las respuestas en los rangos $[0,6],[7,8]$ y $[9,10]$ corresponden a actitudes de usuarios detractores, indiferentes y promotores, respectivamente; el promedio de las respuestas se considera el valor final para el objeto de prueba.

Se llevaron a cabo 5 sesiones de evaluación, en cada una hubo tres participantes y la evaluadora. La duración promedio de cada sesión fue de 40 minutos. Las sesiones se llevaron a cabo en el Laboratorio de Experiencia de Usuario de la UPPue (laboratorio m@ux, http://informatica.uppuebla.edu.mx/ mmedina/maux). Onto4ODS se instaló en tres computadoras personales, se utilizó la versión 71.0.3578.98 del navegador Google Chrome. El análisis de los resultados se realizó a través de sesiones virtuales entre el personal de la UPPue y la UJED. La tabla 5, muestra los valores promedio de los datos recolectados en los cuestionarios previos, (valores en el rango [1, 5], 1 corresponde al mínimo, 5 al máximo).

Tabla 5

Valores previos de los perfiles de participantes

\begin{tabular}{lc}
\hline \multicolumn{1}{c}{ Característica de perfil } & Valor promedio \\
\hline Emplea TIC & 5 \\
Conoce qué es un OA & 3.8 \\
Consulta OAs en línea & 3.2 \\
Interpreta grafos & 4.2 \\
Consulta repositorios de OAs en línea & 3.1 \\
Nivel intermedio o avanzado de inglés & 2.8 \\
\hline
\end{tabular}

Fuente: Elaboración propia.

La tabla 6, muestra los valores del índice SUS normalizados en una escala de 0 a 100 puntos, así como los valores para los índices NPS. 
OBJETO DE APRENDIZAJE PARA LOS ODS EN INGLÉS Y ESPAÑOL CON REPRESENTACIONES SEMÁNTICAS.

Tabla 6

Valores del índice SUS y NPS por participante

\begin{tabular}{lccccc}
\hline Participante & SUS & NPS & Participante & SUS & NPS \\
\hline 1 & 88 & 8 & 9 & 87 & 7 \\
2 & 92 & 9 & 10 & 98 & 7 \\
3 & 87 & 8 & 11 & 88 & 8 \\
4 & 91 & 10 & 12 & 95 & 9 \\
5 & 82 & 10 & 13 & 78 & 7 \\
6 & 89 & 9 & 14 & 84 & 7 \\
7 & 83 & 7 & 15 & 99 & 8 \\
8 & 84 & 8 & Promedio: & $\mathbf{8 8 . 6}$ & $\mathbf{8 . 1}$ \\
\hline
\end{tabular}

Fuente: Elaboración propia.

El resultado promedio obtenido para la escala SUS para Onto4ODS es 88.6, lo cual indica que los participantes no encontraron problemas significativos en su uso y de acuerdo a la interpretación de (Brooke, 2013), se obtuvo un valor para la percepción de uso aceptable. Por otro lado, el índice NPS final fue de 8.1, no hubo usuarios detractores, 3 de 15 participantes mostraron una actitud de indiferencia y 12 serán promotores.

\section{CONCLUSIONES}

El artículo presentó un OA que extrae información del tesauro y las páginas web en inglés y español sobre el DS y los ODS de la ONU y la representa en mapas semánticos, grafos y un modelo semántico. Los términos utilizados para el contenido se encuentran en idioma inglés y español, el objetivo es apoyar la adquisición de un vocabulario común y permitir alcanzar un nivel de conocimientos básico a los estudiantes.

La construcción de este objeto, responde a la problemática identificada por las autoras en esas fuentes de información que afecta a los estudiantes, la cual consiste en que existen diferencias tanto en los aspectos de la interfaz como en la profundidad y nivel de detalle de los contenidos, lo que ocasiona que los estudiantes que sólo revisan los contenidos en español acceden a menor información que la distribuida en inglés. 
OBJETO DE APRENDIZAJE PARA LOS ODS EN INGLÉS Y ESPAÑOL CON REPRESENTACIONES SEMÁNTICAS.

El objeto se construyó con base en el modelo instruccional o metodología ADDIE. En el análisis se establecieron las preguntas generadoras con base en el perfil, habilidades y actitudes de los estudiantes de licenciatura de la UPPue y UJED, también se decidió no incluir en esta primer versión del objeto a ninguno de los indicadores ni sus metas. En el diseño, se trabajó con los elementos pedagógicos del OA como objetivo instruccional, contenido, actividades de aprendizaje y evaluación; así también, se identificaron las tecnologías semánticas y TIC que se utilizarían en el desarrollo e implementación. Se presentaron ejemplos de los contenidos representados como mapas conceptuales, grafos y elementos del modelo semántico. Finalmente, se presentó el estudio exploratorio llevado a cabo en la etapa de evaluación para estimar la percepción de los usuarios potenciales, en el que se obtuvieron valores aceptables para la escala de usabilidad (88.6) y el registro de red de promotores (8.1).

El objeto propuesto representa una aplicación de las tecnologías semánticas y las TICs dirigidas a atender necesidades de formación académica para la sostenibilidad. Como trabajo a futuro, se propone depositar este objeto en el repositorio institucional de la UPPue, de manera que a través del módulo de estadísticas, se obtengan datos sobre su uso a corto y mediano plazo.

\section{REFERENCIAS BIBLIOGRÁFICAS}

Arboleda, S. (2016). El desarrollo sostenible: herramienta para la preservación del medio ambiente como derecho de todos. Producción + Limpia, 11(1), 13-21. Recuperado de http://www.scielo.org.co/pdf/pml/v11n1/v11n1a01.pdf

Branch, R. (2009). Instructional design: the ADDIE approach. Boston: Springer.

Brooke, J. (2013). SUS: a retrospective. Journal of Usability Studies, 8(2), 29-40. Recuperado de http://uxpajournal.org/wp-content/uploads/sites/8/pdf/JUS_Brooke_February_2013.pdf

Brundtland, G., Khalid, M. \& Agnelli, S. (1987). Our common future. Report of the world commission on environment and development. United Nations. Recuperado de http://www.channelingreality.com/Documents/Brundtland_Searchable.pdf

Fessenden, T. (2016). Net promoter score: what a customer-relations metric can tell you about your user experience. Recuperado de https://www.nngroup.com/articles/nps-ux/

Gogus, A. (2012). Bloom's taxonomy of learning objectives. In N. M. Seel (Ed.), Encyclopedia of the sciences of learning. New Yorl: Springer. 
OBJETO DE APRENDIZAJE PARA LOS ODS EN INGLÉS Y ESPAÑOL CON REPRESENTACIONES SEMÁNTICAS.

Novak, J. \& Cañas, A. (2008). The theory underlying concept maps and how to construct and use them.

Recuperado

de

http://cmap.ihmc.us/Publications/ResearchPapers/TheoryUnderlyingConceptMaps.pdf

Chen, A. (2013). Open Access: Open Access at AUT. Recuperado de http://aut.ac.nz.libguides.com/openaccess

Institute of Electrical and Electronics Engineers. (2019). The learning object metadata standard. Recuperado de https://www.ieeeltsc.org/working-groups/wg12LOM/lomDescription/

Muñoz, J., González, J. y Sánchez, J. (2014). La interacción humano-computadora en México. México: Pearson.

Musen, M. (2015). The protégé project: a look back and a look forward. Al Matters, 1(4), 4-12. DOI 10.1145/2757001.2757003

Murillo, J. (2018). Taller de inmersión práctica para educación secundaria mediante aula invertida: Los objetivos de desarrollo sostenible de la agenda 2030 de la ONU. Recuperado de https://prezi.com/p/e3juyjlyfn8y/aula-invertida-ods/

Organización de las Naciones Unidas para la alimentación y la agricultura. (2019). Objetivos de Desarrollo Sostenible. Recuperado de http://www.fao.org/sustainable-developmentgoals/goals/es/

United Nations. (2015). Transforming our world: the 2030 agenda for sustainable development. New York: UN Publishing.

Naciones Unidas México. (2019). Objetivos de Desarrollo Sostenible. Recuperado de http://www.onu.org.mx/agenda-2030/objetivos-del-desarrollo-sostenible/

Suber, P. (2012). Open access. United States: MIT Press Ltd.

United Nations (2019). About the sustainable development goals. https://www.un.org/sustainabledevelopment/sustainable-development-goals/

UNBIS thesaurus. (2019). United Nations Bibliographical Information System. Recuperado de https://lib-thesaurus.un.org/LIB/DHLUNBISThesaurus.nsf/ 